\title{
Labile PT-INR in a Covid-19 Patient Under Long-term Vitamin K Antagonist Therapy: a Case Report
}

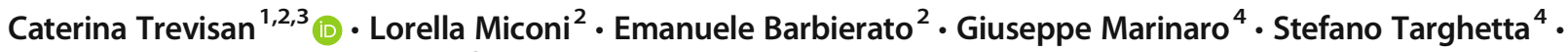 \\ Mario D'agata ${ }^{4}$. Daniela Rinaldi ${ }^{2}$
}

Accepted: 13 August 2020 / Published online: 19 August 2020

(C) Springer Nature Switzerland AG 2020

\begin{abstract}
The COronaVIrus Disease-19 (COVID-19) has been associated with thromboembolic complications, but evidence on its impact on hemorrhagic risk are still scarce. We describe a case of COVID-19 with hemorrhagic complication in the post-acute phase of the disease. The patient was a 93-year-old woman admitted to a post-acute care unit for COVID-19 patients in northern Italy. The patient's medical history included moderate cognitive impairment, atrial fibrillation under oral anticoagulation (warfarin $2.5 \mathrm{mg}$ and $3.75 \mathrm{mg}$ on alternate days, showing stable PT-INR values since October 2018), and arterial hypertension. The patient was affected by COVID-19, treated with supportive therapy. In the post-acute phase of the disease, the patient presented with melena and showed a marked elevation of PT-INR of 25.1. She was treated with venous infusion of three-factor prothrombin complex concentrate and vitamin K until PT-INR reduction. After 5 days, blood analyses revealed normal hemoglobin, PT-INR 2.15, and a slight alteration of liver function. This case supports a strong impact of the infection on the coagulative pattern not only prothrombotically but also by increasing hemorrhagic risk. Special attention should therefore be paid to COVID-19 patients under anticoagulation with vitamin $\mathrm{K}$ antagonist and a shift to heparin treatment until illness resolution may be a safe option in these individuals.
\end{abstract}

Keywords COVID-19 $\cdot$ Oral anticoagulation $\cdot$ Hemorrhagic risk $\cdot$ Case report

\section{Introduction}

The impact of infective diseases on coagulative pattern has been demonstrated by previous studies [1]. This issue concerns, in particular, older individuals who, because of coexisting chronic conditions and pharmacologic treatments, nutritional disorders, and age-related changes in hemostasis, are at higher risk both of thromboembolic and hemorrhagic

Topical Collection on COVID-19

Caterina Trevisan

caterina.trevisan.5@phd.unipd.it

1 Department of Medicine (DIMED), Geriatrics Division, University of Padua, Via Giustiniani 2, 35128 Padua, Italy

2 Ospedale di Comunità, ULSS 6 Euganea, Camposampiero, PD, Italy

3 Aging Research Center, Department of Neurobiology, Care Sciences and Society, Karolinska Institutet and Stockholm University, Stockholm, Sweden

4 Emergency Department, Camposampiero Hospital, ULSS 6 Euganea, Camposampiero, PD, Italy complications [2, 3]. The COronaVIrus Disease-19 (COVID19 ) is caused by SARS-CoV-2, and has been associated with thromboembolic complications [4, 5], such as myocardial infarction and stroke. However, evidence on its impact on hemorrhagic risk are still scarce [6], especially in patients who already are under anticoagulation treatment. We describe a case of COVID-19 with hemorrhagic complication in the post-acute phase of the disease.

\section{Case Presentation}

The patient was a 93-year-old woman admitted to a post-acute care unit for COVID-19 patients in Camposampiero (Padua, northern Italy) on April 10, 2020. The unit was set up by the local health and social services department to offer medium- and long-term care to COVID-19 patients after the acute infection phase. It admits patients who usually have both medical and social needs and its purpose is to alleviate the burden of the pandemic on hospital departments providing higher intensity care. 
The patient was admitted to our unit in the post-acute phase of the disease because of a lack of adequate assistance at home. Despite having moderate cognitive impairment (Short Performance Mental Status Questionnaire score 6/10), the patient lived alone at home and, in the pre-COVID-19 period, she attended a day care center for the elderly and received further assistance from her sons. In addition to cognitive impairment (for which she took trazodone $75 \mathrm{mg}$ /day), her medical history included atrial fibrillation under oral anticoagulation (warfarin $2.5 \mathrm{mg}$ and $3.75 \mathrm{mg}$ on alternate days), and arterial hypertension treated with two diuretics (furosemide $25 \mathrm{mg}$ /day and perindopril $4 \mathrm{mg}$ /day). The patient's PT-INR values had been regularly monitored since October 2018 (time in therapeutic range [TTR] estimated using the traditional method was $71.4 \%$, see Fig. 1).

On March 23, the patient presented with fatigue, high fever, and cough, for which her general practitioner prescribed a nasopharyngeal swab for COVID-19, which was analyzed by reverse transcriptase-polymerase chain reaction (RT-PCR) assay. The test confirmed the presence of severe acute respiratory syndrome coronavirus 2 (SARS-CoV-2) infection and the patient was given supportive therapy with antipyretics. Her symptoms gradually improved from April 7, but since the patient's sons were by then also affected by COVID-19 and were unable to give her assistance, on April 10, the patient was admitted to our unit for post-acute care and assistance.

At admission, the patient was afebrile, her blood pressure and heart rate were within the normal range (BP 120/ $65 \mathrm{mmHg}$, HR $95 \mathrm{bpm}$ arrhythmic), and oxygen saturation in ambient air was $91 \%$. At physical examination, breath sounds were slightly reduced at both lung bases, and there were no fine or coarse crackles. There were no substantial pathologic findings at the abdominal and neurologic examinations. Given that the patient was clinically stable, biochemical analyses were arranged to be carried out a few days later. Meanwhile, her home therapy was continued.
On April 15, the patient presented with melena and BP below $90 / 60 \mathrm{mmHg}$, associated with an HR of $72 \mathrm{bpm}$ and an oxygen saturation in ambient air of $95 \%$. She was transferred to the Emergency Department where recent upper gastrointestinal bleeding was confirmed at nasogastric lavage and digital rectal exam. Blood examination showed mild leukocytosis (WBC $11.2 \times 10^{9} / 1$, with high neutrophil [87.8\%] and low lymphocytes [5.0\%]), normal levels of hemoglobin $(118 \mathrm{~g} / \mathrm{l})$ and platelets $\left(342 \times 10^{9} / \mathrm{l}\right)$, and slightly altered kidney function (estimated GFR $45 \mathrm{ml} / \mathrm{min}$ ). As illustrated in Fig. 1 , the coagulative pattern was markedly altered in respect to trend observed for 18 months before infection, with a PT ratio of 28.71, PT-INR of 25.1, and APTT ratio of 7.62.

The patient was treated with a venous infusion of $2500 \mathrm{UI}$ of three-factor prothrombin complex concentrate (factors II, IX, X) and $10 \mathrm{mg}$ of vitamin K. PT-INR values at blood sampling 2.5 and $11.5 \mathrm{~h}$ after infusion were 2.68 and 1.21, respectively. The hemoglobin concentration after $11.5 \mathrm{~h}$ was $108 \mathrm{~g} / \mathrm{l}$. On April 16, the patient was re-admitted to our unit, anticoagulation was temporarily stopped, and a proton-pump inhibitor (pantoprazol $40 \mathrm{mg}$ ) was administered twice daily via venous infusion. From April 18, the patient no longer exhibited signs of gastrointestinal bleeding. On April 20, blood analyses revealed hemoglobin $117 \mathrm{~g} / \mathrm{l}$, PT-INR 2.15, and a slight alteration of liver function with AST 55 U/1 (normal range: 5-32).

\section{Conclusions}

The temporal relationship between COVID-19 onset and PTINR elevation in a patient whose PT-INR values were stable in the 18 months before the hospitalization supports the hypothesis that this infection may impact on the coagulative pattern not only pro-thrombotically $[4,5]$ but also by increasing hemorrhagic risk. This confirms that special attention
Fig. 1 Patient's PT-INR values during the 18 months before and 1 month after COVID-19. PTINR prothrombin timeinternational normalized ratio, SARS-CoV-2 severe acute respiratory syndrome coronavirus 2

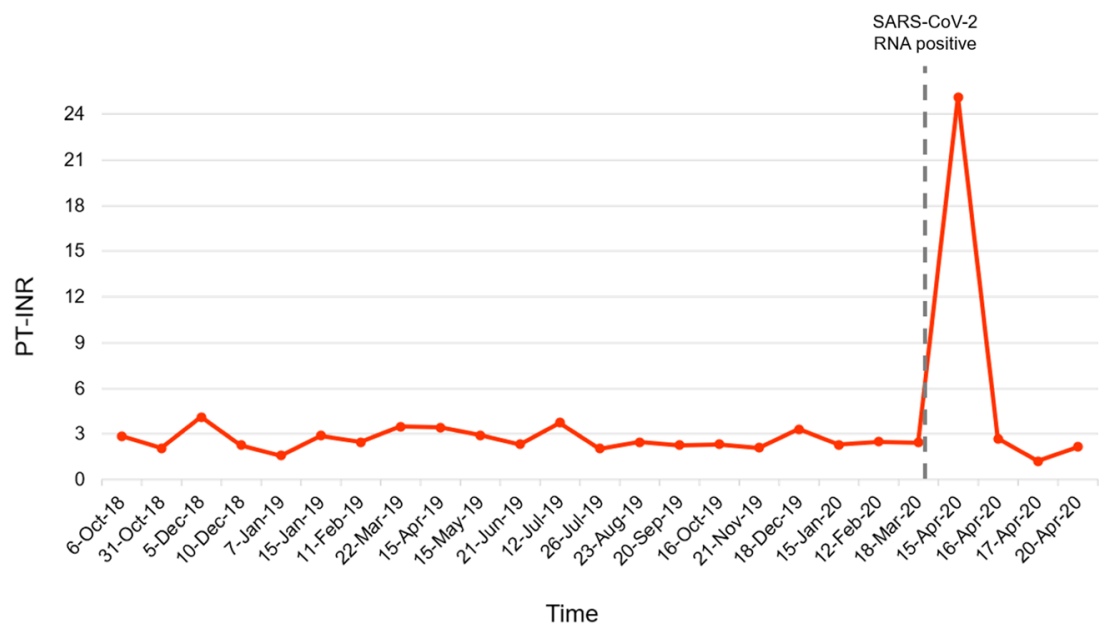

SN Comprehensive Clinical Medicine

a SPRINGER NatURE journal 
should be paid to COVID-19 patients under long-term anticoagulation with vitamin $\mathrm{K}$ antagonist and a shift to heparin treatment until illness resolution may be a safe option in these individuals [7]. Older people may be particularly vulnerable to the influence of COVID-19 on PT-INR instability due to nutritional deficiencies, co-occurring clinical conditions, and possibly liver dysfunction, which may be exacerbated by SARS-CoV-2 infection.

\section{Compliance with Ethical Standards}

Conflict of Interest The authors declare that they have no conflict of interest.

Statement of Human and Animal Rights The study was carried out in accordance with the 1964 Declaration of Helsinki and subsequent amendments or comparable ethical standards.

Consent to Publish Informed consent for reporting the patient's data in an anonymous form was obtained from her next of kin.

\section{References}

1. Goeijenbier M, van Wissen M, van de Weg C, Jong E, Gerdes VEA, Meijers JCM, et al. Review: Viral infections and mechanisms of thrombosis and bleeding. J Med Virol [Internet]. John Wiley \& Sons, Ltd; 2012 [cited 2020 Jul 19];84:1680-96. Available from: http://doi.wiley.com/10.1002/jmv.23354
2. Lenti MV, Pasina L, Cococcia S, Cortesi L, Miceli E, Dominioni CC, et al. Mortality rate and risk factors for gastrointestinal bleeding in elderly patients. Eur J Intern Med [Internet]. Elsevier B.V.; 2019 [cited 2020 Jul 21];61:54-61. Available from: https://doi.org/10. 1016/j.ejim.2018.11.003

3. Parks AL, Fang MC. Anticoagulation in older adults with multimorbidity [Internet]. Clin. Geriatr. Med. W.B. Saunders; 2016 [cited 2020 Jul 21]. p. 331-46. Available from: http://www.geriatric. theclinics.com/article/S0749069016000045/fulltext

4. Klok FA, Kruip MJHA, van der Meer NJM, Arbous MS, Gommers DAMPJ, Kant KM, et al. Incidence of thrombotic complications in critically ill ICU patients with COVID-19. Thromb Res [Internet]. Pergamon; 2020 [cited 2020 Apr 21]; Available from: http://www. ncbi.nlm.nih.gov/pubmed/32291094.

5. Connors JM, Levy JH. COVID-19 and its implications for thrombosis and anticoagulation. Blood [Internet]. NLM (Medline); 2020 [cited 2020 Jul 19];135:2033-40. Available from: https:// ashpublications.org/blood/article-pdf/135/23/2033/1743274/ bloodbld2020006000c.pdf

6. Bao Y, Lin SY, Cheng ZH, Xia J, Sun YP, Zhao Q, et al. Clinical features of COVID-19 in a young man with massive cerebral hemorrhage - case report. SN Compr Clin Med [Internet]. Springer Science and Business Media LLC; 2020 [cited 2020 Jul 21];2:703-9. Available from: https://doi.org/10.1007/s42399-020-00315-y.

7. Testa S, Paoletti O, Giorgi-Pierfranceschi M, Pan A. Switch from oral anticoagulants to parenteral heparin in SARS-CoV-2 hospitalized patients. Intern Emerg Med [Internet]. Springer; 2020 [cited 2020 Apr 21];1-3. Available from: http://www.ncbi.nlm.nih.gov/ pubmed/32297089.

Publisher's Note Springer Nature remains neutral with regard to jurisdictional claims in published maps and institutional affiliations. 